\title{
Spectrally Efficient Constant Envelope Modulation for GNSS Signals
}

\author{
Xuan XIA, Zuping TANG, Jiaolong WEI, Zhihui ZHOU \\ School of Electronic Information and Communications, Huazhong University of Science and Technology, Luoyu Road \\ 1037, 430074 Wuhan, China
}

26329550@qq.com, tang_zuping@hust.edu.cn, jlwei@hust.edu.cn, zhih_zhou@163.com

Submitted January 10, 2018 / Accepted June 20, 2018

\begin{abstract}
As the L-band spectral resources for GNSS services are almost exhausted, new bands such as S-band and $C$-band has been considered for new GNSS signals. In addition to the traditional signal features, out-of-band radiation is an important factor to be considered for signal designing in these new bands. In this paper, a mathematical model for the optimization of GNSS signal waveforms was constructed by integrating ranging accuracy, antijamming performance and out-of-band radiation suppression requirements. In combination with numerical optimization methods, a spectrally efficient constant envelope modulation scheme based on phase trajectory optimization was proposed. The modulated signal has a full-response continuous phase waveform, which facilitates signal processing and implementation at both ends of the transmission and reception. In addition, in the signal design, the balance between signal accuracy, anti-interference performance and out-of-band radiation suppression can be achieved through the adjustment of the weight coefficient. The simulation results show that the out-of-band radiation can be reduced by about 7.2-42.7 dB compared with the existing scheme when the signal performance difference is lower than $2 \mathrm{~dB}$.
\end{abstract}

\section{Keywords}

Spectrally efficient, constant envelope, GNSS, low out-of-band radiation

\section{Introduction}

With the fast increment of global navigation satellite system (GNSS) signals, the contradiction between signal demand and frequency resource restrictions has become increasingly prominent, forcing more attention to be directed toward the spectral efficiency when designing GNSS signals. Currently, the L-band spectral resources for GNSS services are almost exhausted, and thus, new bands, such as S-band and C-band, must be considered for new GNSS signals. At the World Radio Communications Conference in 2000 , the $5010 \mathrm{MHz}$ to $5030 \mathrm{MHz}$ part of the C- band was assigned for Radio Navigation Satellite Services (RNSS) air-to-ground applications, whereas the $5000 \mathrm{MHz}$ to $5010 \mathrm{MHz}$ part of the C-band was assigned for RNSS ground-to-air applications [1]. The adjacent frequency bands for the Radio Astronomy Service (RAS, $4990 \mathrm{MHz}$ to $5000 \mathrm{MHz}$ ) and the Microwave Landing System (MLS, $5030 \mathrm{MHz}-5150 \mathrm{MHz}$ ) have strict restrictions on out-ofband radiation, and the compatibility between uplink GNSS signals and downlink GNSS signals must also be considered [2]. According to the ITU rules, the PSD (normalized power spectral density) of signals in frequency band $4990-5000 \mathrm{MHz}$ cannot exceed $-129.5 \mathrm{~dB} / \mathrm{Hz}$, the PSD of signals in frequency band $5030-5150 \mathrm{MHz}$ cannot exceed $-64.8 \mathrm{~dB} / \mathrm{Hz}$.

GNSS is a typical power-constrained system, and thus, to maximize the power efficiency of the transmitter, the high-power amplifier (HPA) must work at the saturation point. To minimize the nonlinear effects of the HPA, GNSS signals usually adopt constant envelope modulation scheme [3].

There are two ways to reduce out-of-band radiation. One is to design waveforms with lower out-of-band spectral components, and the other is to filter the transmitted signals. Generally, these two methods could both be used in board payload. Relatively speaking, the first method would increase the complexity of the base band signal generation, the second method would increase the complexity of RF channel design. To enhance the out of band suppression ability, we need to use high-order filter, which brings additional insertion loss, larger group delay, and makes the signal waveform quality deteriorated and unstable. Using the first method to design signals with lower out of band radiation can reduce the filter design requirements, which has a positive effect on the quality of the generated navigation signals.

However, given the preconditions of constant envelope characteristics and strict out-of-band radiation, optimizing the performance of the GNSS signals is difficult. The constant envelope modulation signals of L-band (e.g., binary phase-shift keying [BPSK], binary offset carrier [BOC][4], and AltBOC [5]) all feature a rich high-frequency component and low spectrum roll-off speed, which 
are beneficial for optimizing the signal performance. However, these features are not available for the precondition of strict out-of-band radiation. Therefore, the constant envelope continuous phase modulation method is preferred in the design of C-band navigation signals. For example, the Galileo team recommended the use of Gaussian minimum shift keying (GMSK) [6], and Liu et al. proposed minimum shift keying-binary coded signal (MSK-BCS) modulation [7].

This paper proposes a new constant envelope modulation method. Through the joint optimization of the in-phase and quadrature (I\&Q) chip waveform, this modulation method achieves performance equivalent to that of GMSKBPSK(10), GMSK-BOC(5,5), MSK-BCS1 and MSKBCS2. Furthermore, the constant envelope feature and significantly reduced out-of-band radiation of this method indicate that it has better spectral efficiency.

\section{Spectrally Efficient Constant Envelope Modulation (SPECEM)}

SPECEM defines two direct sequence-spread spectrum signal components as follows:

$$
\begin{aligned}
& s_{\mathrm{I}}(t)=\sum_{k=-\infty}^{\infty} a_{k} p_{\mathrm{I}}\left(t-k T_{\mathrm{c}}\right), \\
& s_{\mathrm{Q}}(t)=\sum_{k=-\infty}^{\infty} b_{k} p_{\mathrm{Q}}\left(t-k T_{\mathrm{c}}-d T_{\mathrm{c}}\right)
\end{aligned}
$$

where $a_{k}, b_{k}$ are pseudo-random sequences with values of $\pm 1, T_{\mathrm{c}}$ is the chip width; $p_{\mathrm{I}}(t)$ is the pulse waveform of the in-phase component; $p_{\mathrm{Q}}(t)$ is the pulse waveform of the quadrature-phase component, which are both equal to 0 when $t<0$ or $t \geq T_{\mathrm{c}}$; and $d$ is the pseudo code phase difference between the two signals, $d \in[0,1)$. When the signal pulse waveform of the two signals satisfies the following equations, the combined signal $s(t)=s_{\mathrm{I}}(t)+\mathrm{j} s_{\mathrm{Q}}(t)$ is constantly enveloped.

$$
\left\{\begin{array}{cc}
{\left[p_{\mathrm{I}}(t)\right]^{2}+\left[p_{\mathrm{Q}}\left(t+(1-d) T_{\mathrm{c}}\right)\right]^{2}=c} & 0 \leq t<d T_{\mathrm{c}} \\
{\left[p_{\mathrm{I}}(t)\right]^{2}+\left[p_{\mathrm{Q}}\left(t-d T_{\mathrm{c}}\right)\right]^{2}=c} & d T_{\mathrm{c}} \leq t<T_{\mathrm{c}}
\end{array}\right.
$$

where $c$ is a constant. If these two branch signal code phases are perfectly aligned $(d=0)$, one branch signal or both signals will exhibit a sudden change at the edge of the chip, resulting in a large high-frequency spectral component. Learning from MSK modulation, $d=1 / 2$ is chosen in this paper to reduce the out-of-band spectral component and benefit the symmetry of the chip waveform. Based on the trigonometric functions' feature $\sin ^{2} \theta+\cos ^{2} \theta=1$ and $d=1 / 2$, the following pulse waveform functions are constructed:

$$
\left\{\begin{array}{cc}
p_{\mathrm{I}}(t)=\cos (\theta(t)) & 0 \leq t<T_{\mathrm{c}} / 2 \\
p_{\mathrm{I}}(t)=\cos \left(\theta\left(T_{\mathrm{c}}-t\right)\right) & T_{\mathrm{c}} / 2 \leq t<T_{\mathrm{c}} \\
p_{\mathrm{Q}}(t)=\sin \left(\theta\left(T_{\mathrm{c}} / 2-t\right)\right) & 0 \leq t<T_{\mathrm{c}} / 2 \\
p_{\mathrm{Q}}(t)=\sin \left(\theta\left(t-T_{\mathrm{c}} / 2\right)\right) & T_{\mathrm{c}} / 2 \leq t<T_{\mathrm{c}}
\end{array}\right.
$$

where $\theta(t)$ is a function defined in $\left[0, T_{\mathrm{c}} / 2\right)$. It can be verified that the pulse waveform defined by (3) satisfies the conditions required by (2). Thus, the I\&Q waveform joint optimization problem was transformed into a phase trajectory optimization problem, which is shown as follows.

$$
\left\{\begin{array}{cc}
\max & U[\theta(t)] \\
\text { s.t. } & C[\theta(t)] \leq 0
\end{array}\right.
$$

where $U[\cdot]$ is a utility function that reflects the performance of the modulation signals based on the phase path function $\theta(t) . C[\cdot]$ is a constraint function that reflects the out-of-band radiation and other index values.

The utility function was constructed first. For the GNSS signal, ranging accuracy, anti-jamming performance and anti-multipath performance are critical design factors. Theoretically, the ranging accuracy depends on the code tracking performance, whose lower bound is inversely proportional to the Gabor bandwidth of the modulated signal; literature studies have shown that anti-multipath performance has a similar relationship with the Gabor bandwidth. Therefore, a utility function could be used with the Gabor bandwidth to reflect the ranging accuracy and multipath performance of the modulated signals, as follows:

$$
U_{1}=\int_{-B_{\mathrm{f}} / 2}^{B_{\mathrm{f}} / 2} f^{2} G_{\mathrm{S}}(f) \mathrm{d} f
$$

where $B_{\mathrm{f}}$ is the front-end bandwidth, and $G_{\mathrm{S}}(f)$ is the normalized power spectrum density of the modulated signal $s(t)$. The following equation shows the relationship between $G_{\mathrm{S}}(f)$ and the signal waveform:

$$
G_{\mathrm{S}}(f)=\left(\left|\int_{0}^{T_{\mathrm{c}}} p_{\mathrm{I}}(t) \mathrm{e}^{\mathrm{j} 2 \pi f t} \mathrm{~d} t\right|^{2}+\left|\int_{0}^{T_{\mathrm{c}}} p_{\mathrm{Q}}(t) \mathrm{e}^{\mathrm{j} 2 \pi f t} \mathrm{~d} t\right|^{2}\right) / T_{\mathrm{c}}
$$

where $p_{\mathrm{I}}(t)$ and $p_{\mathrm{Q}}(t)$ can be derived from (3).

The anti-jamming performance is much more complicated to evaluate. This phenomenon is related to different processing steps, such as acquisition, tracking, and demodulation, and different types of jamming. Xue et al. proposed a set of indicators, and evaluation of the anti-jamming performance can be constructed based on these indicators [8]. In fact, under the assumptions of long code, these indicators exhibit the same trend when they are influenced by signal modulation, and selecting representative indicators can simplify the waveform optimization process. The anti-matched-spectrum-jamming merit factor $Q_{\text {DemAJMS }}$ 
was adopted to measure the modulation anti-jamming performance. The anti-matched-spectrum-jamming merit factor $Q_{\text {DemAJMS }}$ is defined as:

$$
Q_{\text {DemAJMS }}=10 \times \log _{10}\left(\frac{1}{R_{\mathrm{d}} \times \int_{-B_{\mathrm{f}} / 2}^{B_{f} / 2} G_{\mathrm{S}}^{2}(f) \mathrm{d} f}\right)(\mathrm{dB})
$$

where $R_{\mathrm{d}}$ denotes the message rate.

Therefore, the performance of the anti-jamming utility function can be defined as:

$$
U_{2}=1 / \int_{-B_{\mathrm{f}} / 2}^{B_{\mathrm{f}} / 2} G_{\mathrm{S}}^{2}(f) \mathrm{d} f .
$$

In summary, the integrated utility function of a GNSS signal is as follows:

$$
U=\log _{10} U_{1}+\omega \log _{10} U_{2}
$$

where $\omega$ is the weighting factor of the anti-jamming performance and anti-multipath performance with respect to the ranging performance. Applying the logarithmic operation can avoid the problems caused by different scales.

The design of the constraint function is related to the out-of-band radiation limitation of the frequency band in which the signal is located, assuming that $N$ adjacent frequency bands of the GNSS signal are involved, that the frequency range of the $i$-th frequency band is $\left[f_{L}^{i}, f_{U}^{i}\right]$, the out-of-band radiation constraints are constructed as follows

$$
C_{i}=\frac{\int_{f_{L}^{i}}^{f_{U}^{i}} G_{\mathrm{S}}(f) \mathrm{d} f}{f_{U}^{i}-f_{L}^{i}}-I_{i}
$$

where $1 \leq i \leq N, I_{i}$ is the maximum average $\operatorname{PSD}$ (APSD) in the $i$-th frequency band derived from ITU rules.

\section{Numerical Optimization Method}

First, the signal waveform is sampled; the sampling rate $f_{\mathrm{s}}=2 K R_{\mathrm{c}}, K$ is an integer, and $R_{\mathrm{c}}$ is the code rate. Then, the phase trajectory samples to be optimized can be expressed as a vector

$$
\boldsymbol{\theta}=\left[\theta_{1}, \theta_{2}, \ldots, \theta_{K}\right] .
$$

The waveform sampling values of the I\&Q branch signals defined in (3) are as follows:

$$
p_{\mathrm{I}}=\cos \left(\left[\boldsymbol{\theta}, \boldsymbol{\theta}_{\mathrm{f}}\right]^{\mathrm{T}}\right), p_{\mathrm{Q}}=\sin \left(\left[\boldsymbol{\theta}_{\mathrm{f}}, \boldsymbol{\theta}\right]^{\mathrm{T}}\right)
$$

where $[\cdot]^{\mathrm{T}}$ denotes the matrix transpose. $\boldsymbol{\theta}_{\mathrm{f}}$ is the flipped vector of $\boldsymbol{\theta}$, which is defined as $\boldsymbol{\theta}_{\mathrm{f}}=\left[\theta_{K}, \theta_{K-1}, \ldots, \theta_{1}\right]$. A frequency vector is defined as follows:

$$
\mathbf{f}=[-K M,-K M+1, \ldots, K M-1]^{\mathrm{T}} \frac{R_{\mathrm{c}}}{M}
$$

where $M$ (an integer) denotes the integer multiple of the oversampling power spectrum. The power spectrum vector could be calculated as

$$
\mathbf{S}=\frac{\operatorname{Diag}\left[\left(\mathbf{V} \cdot \mathbf{p}_{\mathbf{I}}\right)\left(\mathbf{V} \cdot \mathbf{p}_{\mathbf{I}}\right)^{\prime}\right]+\operatorname{Diag}\left[\left(\mathbf{V} \cdot \mathbf{p}_{\mathbf{Q}}\right)\left(\mathbf{V} \cdot \mathbf{p}_{\mathbf{Q}}\right)^{\prime}\right]}{4 K^{2} R_{\mathrm{c}}}
$$

where $(\cdot)^{\prime}$ denotes conjugate transposition, Diag[·] means extracting the main diagonal elements, and $\mathbf{S}$ is a column vector. $\mathbf{V}$ is a vandermonde matrix with $2 K M$ rows and $2 K$ columns that is constructed by vector $\exp \left[\mathrm{j} 2 \pi \mathbf{f} T_{\mathrm{c}} /(2 K)\right]$, which is defined as:

$$
\boldsymbol{V}=\left[1, \mathrm{e}^{\mathrm{j} \frac{\pi T_{c}}{K} f},\left(\mathrm{e}^{\mathrm{j} \frac{\pi T_{\mathrm{c}}}{K} f}\right)^{2}, \ldots,\left(\mathrm{e}^{\mathrm{j} \frac{\pi T_{\mathrm{c}}}{K} f}\right)^{2 K-1}\right] .
$$

The above process may also be implemented on a zero-padded fast Fourier transform (FFT). The corresponding utility function is:

$U_{1}=\sum_{m=m_{1}}^{m_{2}}(m-K M-1)^{2} S_{m}\left(\frac{R_{\mathrm{c}}}{M}\right)^{3}, \quad U_{2}=\left(\sum_{m=m_{1}}^{m_{2}} S_{m}\right)^{-1} \frac{M}{R_{\mathrm{c}}}$

where $S_{m}$ is the $m$-th element of vector $\mathbf{S}$,

$$
m_{1}=\left\lfloor\frac{K R_{\mathrm{c}}-B_{\mathrm{f}} / 2}{R_{\mathrm{c}} / M}\right\rfloor+1, \quad m_{2}=\left\lfloor\frac{K R_{\mathrm{c}}+B_{\mathrm{f}} / 2}{R_{\mathrm{c}} / M}\right\rfloor+1
$$

where $\lfloor\cdot\rfloor$ means rounding down. By definition, the utility function of the discrete waveform is $U=$

$\log \left(\sum_{m=m_{1}}^{m_{2}}(m-K M-1)^{2} S_{m}\right)-\omega \log \left(\sum_{m=m_{1}}^{m_{2}} S_{m}{ }^{2}\right)+\log \left(\frac{R_{\mathrm{c}}}{M}\right)^{3-\omega}$.

Note that, a normal letter $U$ is used to express the utility function of the discrete waveform, and thereby distinguish it from the utility function of the continuous waveform.

Considering the monotonicity of the $\log$ function, and assuming that $R_{\mathrm{c}}, M$, and $\omega$ are constants, so $\log \left(R_{\mathrm{c}} / M\right)^{3-\omega}$ is a constant, in the process of searching the maximum of (18), we could ignore it. The phase path optimization can be expressed as

$$
\begin{aligned}
& \arg \max _{\theta} U= \\
& \arg \max _{\theta}\left[\log \left(\sum_{m=m_{1}}^{m_{2}}(m-K M-1)^{2} S_{m}\right)-\omega \log \left(\sum_{m=m_{1}}^{m_{2}} S_{m}{ }^{2}\right)\right]
\end{aligned}
$$

with the following restrictions: 


$$
\left\{\begin{array}{c}
C_{i}=\sum_{m=m_{L}^{i}}^{m_{U}^{i}} S_{m} \frac{R_{\mathrm{c}}}{M}-I_{i} \\
m_{L}^{i}=\left\lfloor\frac{K R_{\mathrm{c}}+f_{L}^{i}-f_{\mathrm{c}}}{R_{\mathrm{c}} / M}\right\rfloor+1, m_{U}^{i}=\left\lfloor\frac{K R_{\mathrm{c}}+f_{U}^{i}-f_{\mathrm{c}}}{R_{\mathrm{c}} / M}\right\rfloor+1
\end{array}\right.
$$

This optimization problem may be solved by any techniques in optimization theory. In this paper, the penalty function method is adopted. The penalty function technique converts the constrained optimization into an approximate equivalent but unconstrained search of the objective function. The unconstrained optimization problem is minimizing the following

$$
F(\theta)=-\mathrm{U}(\theta)+\sum_{i=1}^{N} r_{i} \cdot \mathrm{C}_{i} .
$$

Here we use the Quasi-Newton method to get the optimal result of the optimization problem. $r_{i}$ is the penalty factor of out-of-band suppression.

\section{Simulation Results}

In this section, using the C-band as an example and an RNSS air-to-ground application located at 5010 to $5030 \mathrm{MHz}$, the mainly out-of-band restrictions come from the RAS band, and the compatibility between the RNSS downlink signal and the RNSS uplink signal must be considered. In the optimization process, the I\&Q branch signals are staggered by half a chip to satisfy the constant envelope feature, and the code rate is $1 \mathrm{MHz}$. To obtain a balance between bandwidth limitation and performance improvement, the penalty factor $r$ is varied. $r=1 \mathrm{e} 5$ is set to find the optimized waveform in Fig. 1, and the weighting factor $\omega=1$.

Figure 2 shows the PSDs of the proposed SPECEM, GMSK-BPSK(10), GMSK-BOC(5,5), MSK-BCK1 and MSK-BCS2 methods, where MSK-BCS1 means MSK$\operatorname{BCS}([1,1,1,1,-1,-1,1,-1,1,-1], 1)$, and MSK-BCS2 means MSK-BCS([1,-1,1,-1,1,-1,1,-1,1,1],1) [7]. According to Fig. 2, the proposed SPECEM method has an obvious main lobe in the RNSS downlink band and much lower APSD than the other modulations in adjacent bands. Thus, this method has less out-of-band radiation and better compatibility with the RAS and RNSS uplink bands.

In Tab. 1 the Gabor bandwidth, $Q_{\text {DemAJMS }}$, RAS band APSD and RNSS uplink band APSD of SPECEM and other modulations are compared.

The Gabor bandwidth is the root-mean-square (RMS) bandwidth in hertz [8], and as the Gabor bandwidth increases, the inherent ability to avoid small code tracking errors improves. It is defined as

$$
\beta_{\text {Gabor }}=\sqrt{\int_{-B_{\mathrm{f}} / 2}^{B_{\mathrm{f}} / 2} f^{2} G_{s}(f) \mathrm{d} f} .
$$

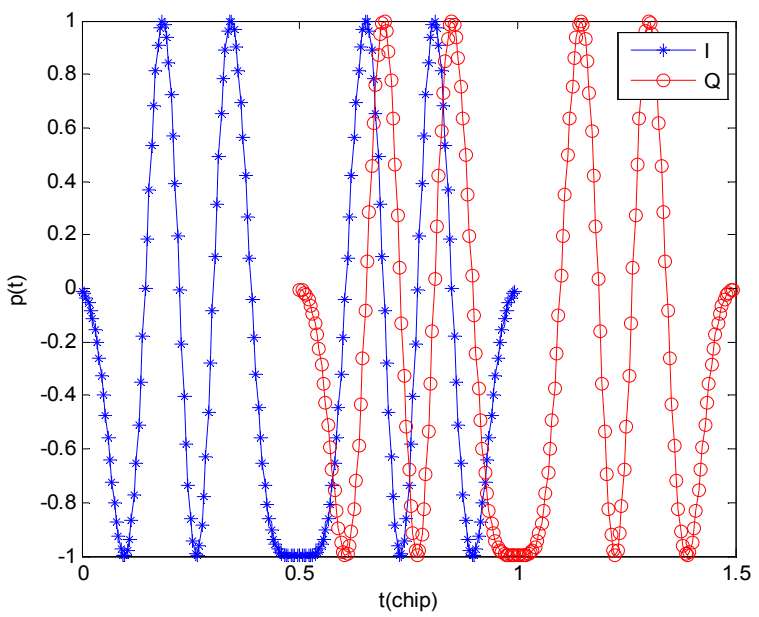

Fig. 1. Optimized waveform.

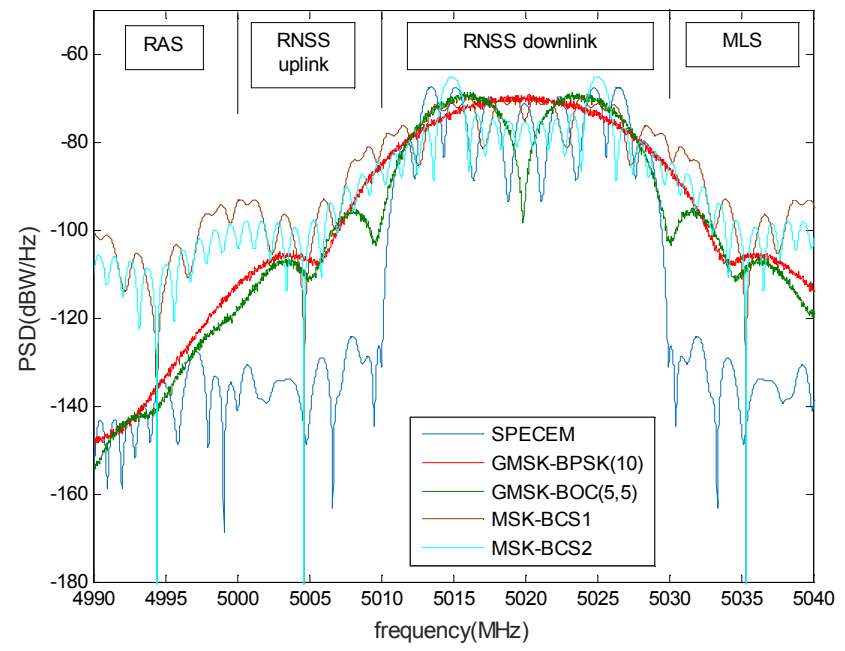

Fig. 2. Signal option power spectra.

\begin{tabular}{|l|c|c|c|c|}
\hline $\begin{array}{l}\text { Signal } \\
\text { option }\end{array}$ & $\begin{array}{c}\text { Gabor } \\
\text { bandwidth } \\
(\mathbf{d B M H z})\end{array}$ & $\begin{array}{c}Q_{\text {DemAJMS }} \\
\text { (dB) }\end{array}$ & $\begin{array}{c}\text { RAS Band } \\
\text { APSD } \\
(\mathbf{d B W} / \mathbf{H z})\end{array}$ & $\begin{array}{c}\text { Uplink Band } \\
\text { APSD } \\
(\mathbf{d B W} / \mathbf{H z})\end{array}$ \\
\hline SPECEM & 14.1 & 52.4 & -135.8 & -131.4 \\
\hline $\begin{array}{l}\text { GMSK- } \\
\text { BPSK(10) }\end{array}$ & 11.2 & 54.2 & -123.2 & -96.0 \\
\hline $\begin{array}{l}\text { GMSK- } \\
\text { BOC(5,5) }\end{array}$ & 12.9 & 53.9 & -128.2 & -102.2 \\
\hline MSK-BCS1 & 13.1 & 54.3 & -100.6 & -88.7 \\
\hline MSK-BCS2 & 13.8 & 50.2 & -105.3 & -93.4 \\
\hline
\end{tabular}

Tab. 1. The indicators of the signal options.

Table 1 shows that the Gabor bandwidth of SPECEM is larger than GMSK-BPSK(10), GMSK-BOC $(5,5)$, MSK-BCS1, and MSK-BCS2. SPECEM has a $2.9 \mathrm{dBMHz}$ advantage compared to GMSK-BPSK(10). GMSK-BOC $(5,5)$, MSK-BCS1 and MSK-BCS2's Gabor bandwidth are $12.9 \mathrm{dBMHz}, 13.0 \mathrm{dBMHz}, 13.8 \mathrm{dBMHz}$, respectively.

$Q_{\text {DemAJMs }}$ evaluates the anti-interference performance of the GNSS signal, and larger values of this index reflect better signals. In Tab. 1 , the $Q_{\text {DemAJMS }}$ of SPECEM is slightly lower than those of GMSK-BPSK(10), GMSK$\operatorname{BOC}(5,5)$ and MSK-BCS but slightly higher than that of MSK-BCS2. 
To measure SPECEM's compatibility with the RAS band, their APSDs were compared. The APSD of SPECEM is $-135.8 \mathrm{dBW} / \mathrm{Hz}$, while the APSDs of GMSKBPSK(10), GMSK-BOC $(5,5)$, MSK-BCS1 and MSK$\mathrm{BCS} 2$ are $-123.2 \mathrm{dBW} / \mathrm{Hz}, \quad-128.2 \mathrm{dBW} / \mathrm{Hz}$, $-100.6 \mathrm{dBW} / \mathrm{Hz}$, and $-105.3 \mathrm{dBW} / \mathrm{Hz}$ respectively. Thus, SPECEM achieves an improvement of $7.2-35.6 \mathrm{dBW} / \mathrm{Hz}$ compared to the other modulations. Similarly, for RNSS uplink signals, the APSD of SPECEM is $-131.4 \mathrm{dBW} / \mathrm{Hz}$, while those of GMSK-BPSK(10), GMSK-BOC $(5,5)$, MSK-BCS1 and MSK-BCS2 are $-96 \mathrm{dBW} / \mathrm{Hz}$, $-102.2 \mathrm{dBW} / \mathrm{Hz}, \quad-88.7 \mathrm{dBW} / \mathrm{Hz}$ and $-93.4 \mathrm{dBW} / \mathrm{Hz}$, respectively. Therefore, SPECEM achieves an improvement of $29.2-42.7 \mathrm{dBW} / \mathrm{Hz}$ compared to the other modulations. These lower APSD values allow the C-band to transmit power and reduce the influence of the C-band free space loss growth, thereby decreasing the complexity of the on-board load required.

The multipath error envelope and mean multipath error are used to reflect the multipath performance. Figure 3

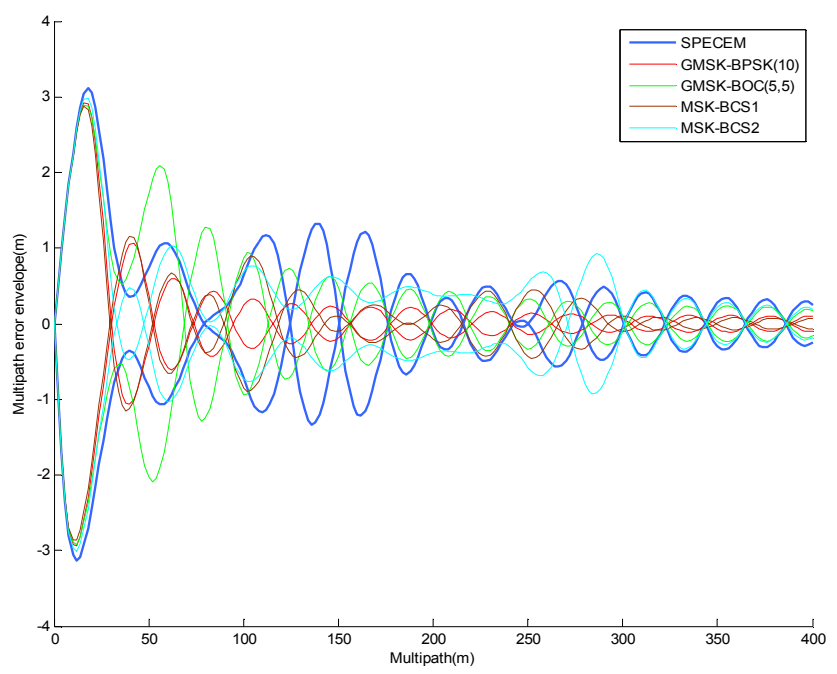

Fig. 3. Multipath error envelope.

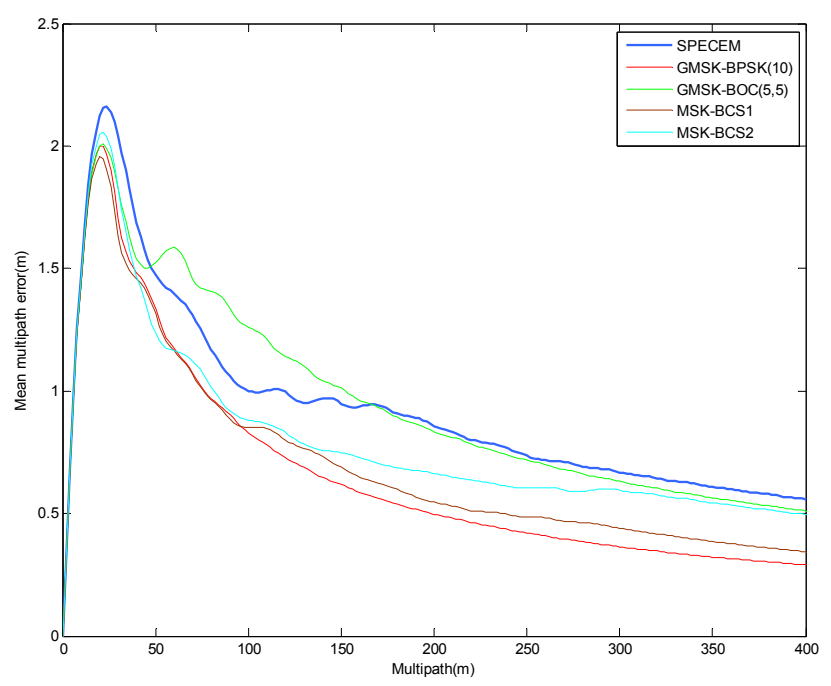

Fig. 4. Mean multipath error. shows that the multipath error envelope of SPECEM is slightly larger than those of the other modulations when the multipath delay is located in $(100,200)$. In contrast, when the multipath delay is located in $(0,100)$ and $(200,400)$, SPECEM's performance is equal to those of the other modulations. Figure 4 presents the mean multipath errors of the modulations; all the modulations exhibit similar performance in terms of this value.

\section{Conclusion}

In this paper, a mathematical model for the optimization of GNSS signal waveforms was constructed by integrating ranging accuracy, anti-jamming performance and out-of-band radiation suppression requirements. By adjusting the parameters $\omega$ and $r$, the balance between signal accuracy, anti-interference performance and out-of-band radiation suppression could be achieved. The simulation results show that the out-of-band radiation can be reduced by about $7.2-42.7 \mathrm{~dB}$ compared with the existing scheme when the signal performance difference is lower than $2 \mathrm{~dB}$.

\section{Acknowledgments}

This work is supported by the National Science Foundation of China (NSFC), Grant 61401171.

\section{References}

[1] IRSIGLER, M., HEIN, G. W., PEIFFER, A. S. Use of C-band frequencies for satellite navigation: benefits and drawbacks. GPS Solutions, 2004, vol. 8, no. 3, p. 119-139. DOI: 10.1007/s10291004-0098-2

[2] XUE, R., CAO, Q. M., WEI, Q. A flexible modulation scheme design for C-band GNSS signals. Mathematical Problems in Engineering, 2015, article ID 165097, p. 1-8. DOI: $10.1155 / 2015 / 165097$

[3] HUANG, X., ZHU, X., OU, G. Constant-envelope dual QPSK-like modulation and its generalised form for modern GNSS signals. Electronics Letters, 2015, vol. 51, no. 2, p. 175-177. DOI: 10.1049/EL.2014.3487

[4] BETZ, J. W. Binary offset carrier modulations for radionavigation. Navigation: Journal of The Institute of Navigation, 2001, vol. 48, no. 4, p. 227-246. DOI: 10.1002/J.2161-4296.2001.TB00247.X

[5] LAURENT, L., ARTAUD, G., ISSLER, J. AltBOC for dummies or everything you always wanted to know about AltBOC. In Proceedings of the 21st International Technical Meeting of the Satellite Division of The Institute of Navigation (ION GNSS 2008). Savannah (USA), Sep. 2008, p. 961-970.

[6] AVILA-RODRIGUEZ, J. A., WALLNER, S., WON, J. H., et al. Study on a Galileo signal and service plan for c-band. In Proceedings of the 21st International Technical Meeting of the Satellite Division of The Institute of Navigation (ION GNSS 2008). Savannah (USA), Sep. 2008, p. 2515-2529.

[7] LIU, M., ZHAN, X., LI, W., JING, S., CHEN, M. An improved MSK-BCS modulation for global navigation satellite systems in $\mathrm{C}$ 
band. IEEJ Transactions on Electrical and Electronic Engineering, 2016, vol. 11, no. 4, p. 474-479. DOI: $10.1002 /$ tee. 22264

[8] XUE, R., SUN, Y., ZHAO, D. CPM signals for satellite navigation in the S and C bands. Sensors, 2015, vol. 15, no. 6, p. 13184 to 13200. DOI: $10.3390 / \mathrm{s} 150613184$

\begin{abstract}
About the Authors ...
Xuan XIA was born in Hubei, China in 1987. He received his B.S. from Huazhong University of Science and Technology in 2009. He is currently working toward the Ph.D. degree at the School of Electronic Information and Communications, Huazhong University of Science and Technology, China. His research interests include next generation GNSS signals and signal processing algorithm of GNSS receivers.

Zuping TANG (corresponding author) was born in Chongqing, China in 1981. He received his B.S., M.Sc. and Ph.D. degrees from Huazhong University of Science and Technology, Wuhan, China, in 2002, 2005 and 2009,
\end{abstract}

respectively. He is currently an associate professor of the School of Electronic Information and Communications, Huazhong University of Science and Technology. His research interests include GNSS signal design theory, signal quality evaluation and GNSS receiver technique.

Jiaolong WEI was born in Jiangxi, China in 1965. He received his B.S., M.Sc. and Ph.D. degrees from Huazhong University of Science and Technology, Wuhan, China, in 1986, 1990 and 2004, respectively. He is currently a professor, Ph.D. supervisor and vice-dean of the School of Electronic Information and Communications, Huazhong University of Science and Technology. His research interests include wireless communications and networks, intelligence computation and satellite navigation.

Zhihui ZHOU was born in Hunan, China in 1988. He received his B.S. from Huazhong University of Science and Technology in 2011. He is currently working toward the Ph.D. degree at the School of Electronic Information and Communications, Huazhong University of Science and Technology, China. His research interests include next generation GNSS signals and signal processing algorithm of GNSS receivers. 